Case Report

\title{
Esophageal Perforation with Unilateral Fluidothorax Caused by Nasogastric Tube
}

\author{
Lukas P. Mileder, ${ }^{1}$ Martin Müller, ${ }^{1}$ Friedrich Reiterer, ${ }^{1}$ Alexander Pilhatsch, ${ }^{2}$ \\ Barbara Gürtl-Lackner, ${ }^{3,4}$ Berndt Urlesberger, ${ }^{1}$ and Wolfgang Raith ${ }^{1}$ \\ ${ }^{1}$ Division of Neonatology, Department of Pediatrics and Adolescent Medicine, Medical University of Graz, Graz, Austria \\ ${ }^{2}$ Division of Pediatric Radiology, Department of Radiology, Medical University of Graz, Graz, Austria \\ ${ }^{3}$ Institute of Pathology, Medical University of Graz, Graz, Austria \\ ${ }^{4}$ Department of Pathology, Skåne Regional and University Laboratories, Lund, Sweden
}

Correspondence should be addressed to Lukas P. Mileder; lukas.mileder@medunigraz.at

Received 29 July 2016; Accepted 24 August 2016

Academic Editor: Ian J. Griffin

Copyright (C) 2016 Lukas P. Mileder et al. This is an open access article distributed under the Creative Commons Attribution License, which permits unrestricted use, distribution, and reproduction in any medium, provided the original work is properly cited.

Preterm infants are highly susceptible to injuries following necessary and often life-saving medical interventions. Esophageal perforation is a rare, yet serious complication that can be caused by aerodigestive tract suction, endotracheal intubation, or nasogastric tube placement. We present the case of a neonate born at 23 weeks plus three days of gestation with chest radiography showing malposition of the nasogastric feeding tube and massive right-sided effusion of Iopamidol in the pleural cavity due to esophageal perforation. In addition, the article summarizes common signs and symptoms associated with esophageal perforation in infants and discusses diagnostic approaches.

\section{Introduction}

Esophageal perforation (EP) is a rare, yet serious complication [1], with an incidence of 1:124 among preterm infants weighing less than $1500 \mathrm{~g}$ at birth [2]. Mortality is as high as $20 \%$ in these vulnerable patients [3].

\section{Case Presentation}

A female twin neonate born at 23 weeks plus three days of gestation with a birth weight of $538 \mathrm{~g}$ was intubated after birth due to respiratory distress and received surfactant repeatedly. Because of signs of evolving bronchopulmonary dysplasia and pulmonary hypertension, she was treated with hydrocortisone and nitric oxide. Transcranial sonography revealed grade II intraventricular hemorrhage.

On day 8 of life, feeding problems with bilious gastric residuals, constipation, abdominal distension, and lactic acidosis developed, which were initially managed conservatively. Spontaneous ileum perforation necessitated laparoscopic surgery, including local bowel resection and creation of an ileostomy, on day 12. One day after surgery, clinical signs suggested development of an intestinal volvulus. After injection of Iopamidol for evaluation of intestinal obstruction, chest radiography showed massive right-sided effusion in the pleural cavity and malposition of the nasogastric feeding tube (Figure 1). Pleural drainage resulted in prompt stabilization of vital parameters after extraction of $10 \mathrm{~mL}$ of serous liquid. Nitric oxide therapy was gradually reduced and could be abandoned on day 13 .

Due to numerous morbidities including intraventricular hemorrhage and arterial hypotension despite inotropic support, the neonate died on day 20. Autopsy showed regular esophageal development with minimal inflammatory reaction below the muscular layer and found lung immaturity to be the cause of death.

\section{Discussion}

Iatrogenic EP can be caused by vigorous pharyngoesophageal suction, traumatic endotracheal intubation, and insertion of 


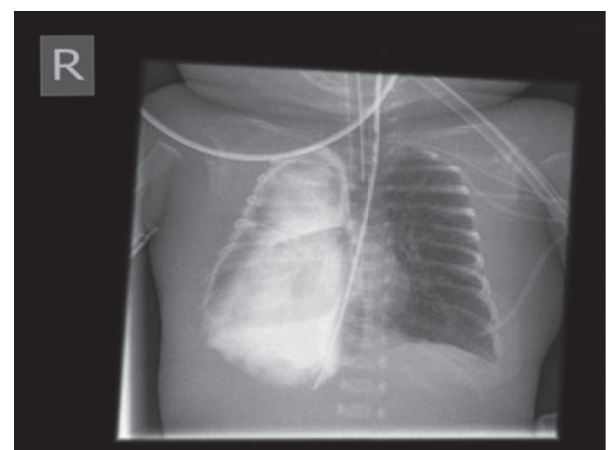

FIGURE 1: Chest radiograph after contrast agent application showing massive effusion in the pleural cavity and abnormal right-sided position of the nasogastric tube.

naso-/orogastric feeding tubes [4]. Clinical signs and symptoms of EP include inability to pass a gastric tube, feeding difficulties, bloody oral secretions, fever, cyanosis, and subcutaneous emphysema $[4,5]$. In our case, EP did not present with sudden respiratory deterioration, which is the most common symptom [1]. Apparently, there is a wide spectrum of the clinical presentation of EP especially in extremely preterm infants, including malposition of the nasogastric feeding tube.

Diagnosis of EP is based on clinical findings, chest radiography, and endoscopy [3]. Radiographic evidence of EP includes pneumothorax, pneumomediastinum, or subcutaneous air [4], with proximal EP usually causing left-sided abnormalities and distal perforations commonly appearing on the right side [6]. Flexible endoscopy has been shown to allow for diagnosis and precise location of EP at the bedside while avoiding contrast use and radiation exposure [7] and should therefore be considered as diagnostic measure especially in preterm infants with clinical signs of EP.

\section{Competing Interests}

The authors declare that there is no conflict of interest regarding the publication of this paper.

\section{Authors' Contributions}

Friedrich Reiterer, Wolfgang Raith, Martin Müller, Berndt Urlesberger, and Alexander Pilhatsch were involved in clinical management of the patient. Barbara Gürtl-Lackner performed postmortem autopsy. Lukas P. Mileder and Wolfgang Raith acquired patient consent. Lukas P. Mileder and Martin Müller drafted the manuscript. All authors critically revised it for important intellectual content and approved the final version before submission.

\section{References}

[1] P. S. Shah, M. S. Dunn, and V. S. Shah, "Oesophageal perforation in preterm neonates: not an innocent bystander," Journal of Paediatrics and Child Health, vol. 39, no. 9, pp. 697-699, 2003.
[2] L. Filippi, M. Pezzati, and C. Poggi, "Use of polyvinyl feeding tubes and iatrogenic pharyngo-oesophageal perforation in very-low-birthweight infants," Acta Paediatrica, vol. 94, no. 12, pp. 1825-1828, 2005.

[3] E. Sapin, L. Gumpert, A. Bonnard et al., "Iatrogenic pharyngoesophageal perforation in premature infants," European Journal of Pediatric Surgery, vol. 10, no. 2, pp. 83-87, 2000.

[4] N. Sudhakaran and C. P. Kirby, "Pitfalls of gastric intubation in premature infants," Journal of Paediatrics and Child Health, vol. 37, no. 2, pp. 195-197, 2001.

[5] S. A. Engum, J. L. Grosfeld, K. W. West, F. J. Rescorla, L. R. Scherer, and W. G. Vaughan, "Improved survival in children with esophageal perforation," Archives of Surgery, vol. 131, no. 6, pp. 604-611, 1996.

[6] E. Panieri, A. J. W. Millar, H. Rode, R. A. Brown, and S. Cywes, "Iatrogenic esophageal perforation in children: patterns of injury, presentation, management, and outcome," Journal of Pediatric Surgery, vol. 31, no. 7, pp. 890-895, 1996.

[7] W.-J. Soong, "Endoscopic diagnosis and management of iatrogenic cervical esophageal perforation in extremely premature infants," Journal of the Chinese Medical Association, vol. 70, no. 4, pp. 171-175, 2007. 


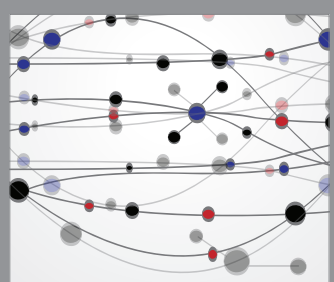

The Scientific World Journal
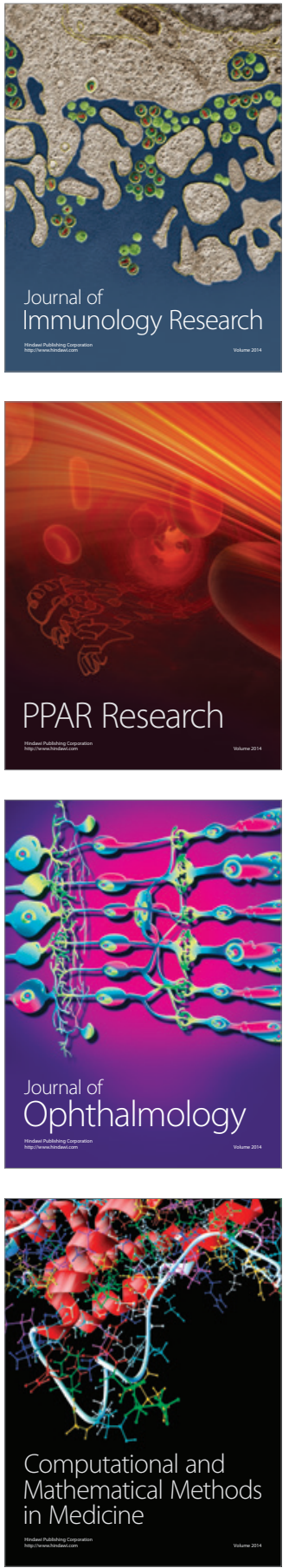

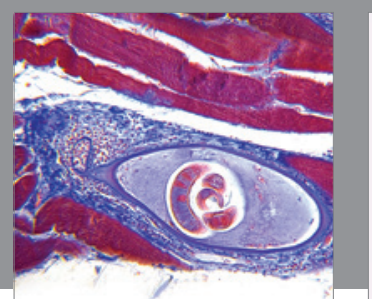

Gastroenterology Research and Practice

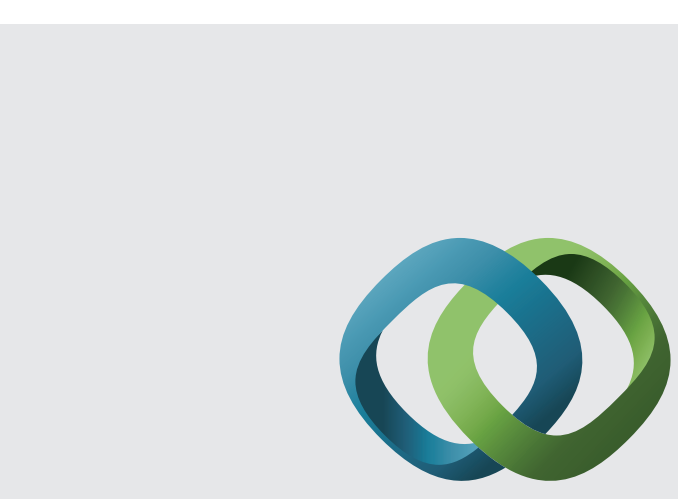

\section{Hindawi}

Submit your manuscripts at

http://www.hindawi.com
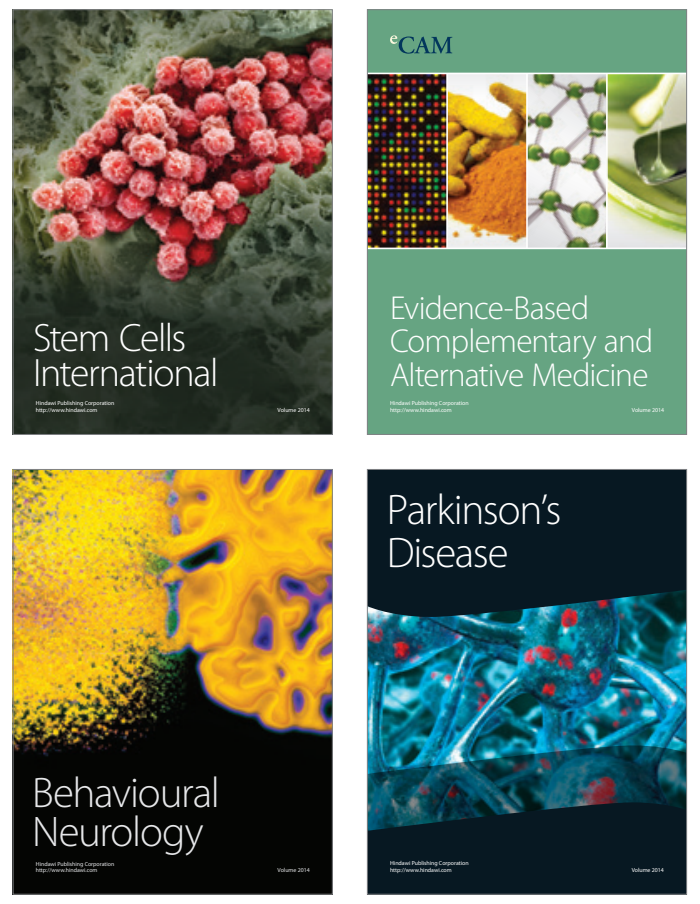
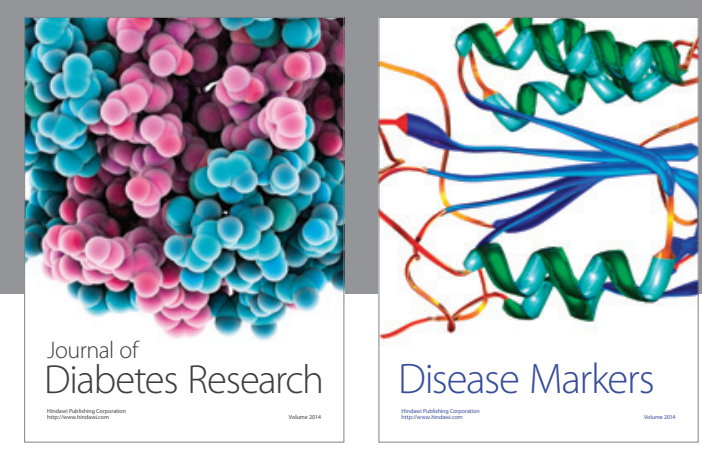

Disease Markers
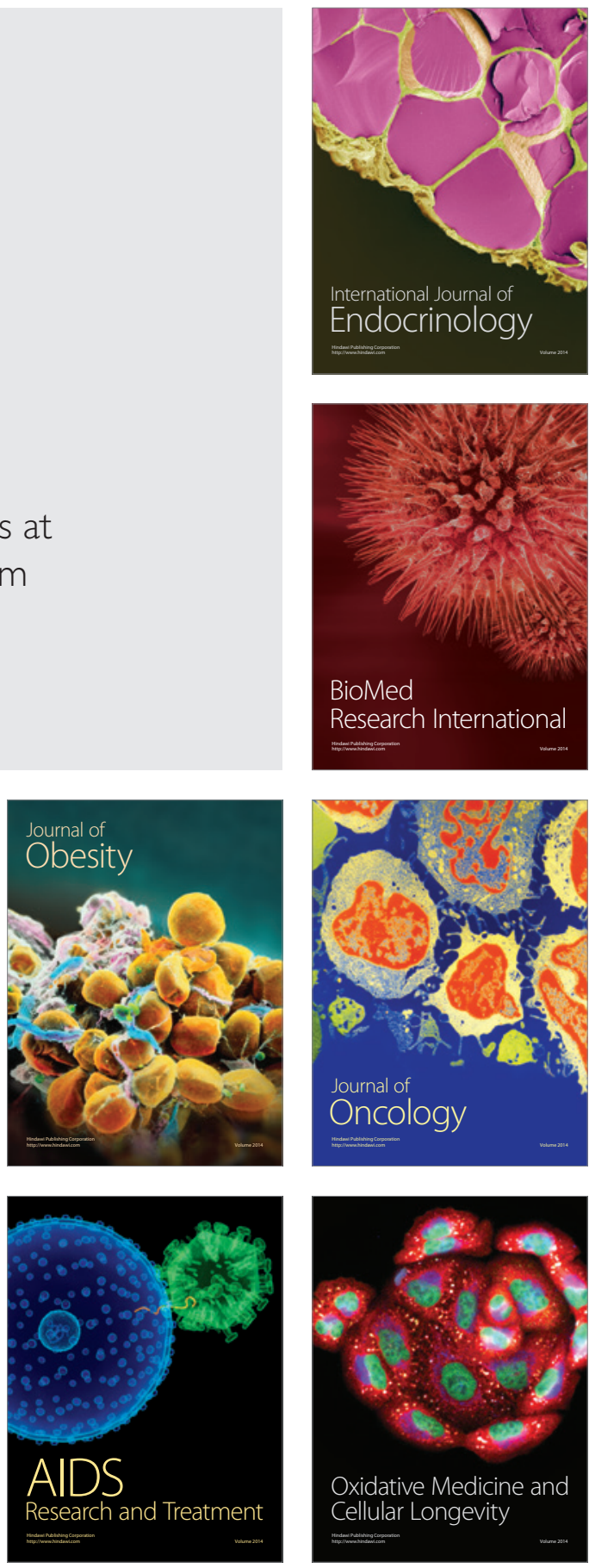